Available online at: http://ijmt.ir/browse.php?a code=A-10-990-1\&sid=1\&s/c lang=en

\title{
Transient Behavior of Saltwater Wedge and Mixing Zone in Head- Controlled Coastal Aquifer: Experimental Measurements and Numerical Modeling
}

\author{
Abbasali Rezapour ${ }^{1 *}$, Seyed Fazlolah Saghravani ${ }^{2}$, Alireza Ahmadyfard ${ }^{3}$, Mehdi Rezapour ${ }^{4}$ \\ ${ }^{1}$ Department of Civil Engineering, Birjand University of Technology; email: \\ abbas.rezapoor@birjandut.ac.ir \\ ${ }^{2}$ Department of Civil Engineering, Shahrood University of Technology \\ ${ }^{3}$ Department of Electrical Engineering, Shahrood University of Technology \\ ${ }^{4}$ Department of Civil Engineering, Chabahar Maritime University
}

\section{ARTICLE INFO}

Article History:

Received: 6 Dec. 2018

Accepted: 21 Feb. 2019

\section{Keywords:}

saltwater intrusion

transient condition

saltwater wedge

mixing zone

image processing

numerical modelling

\begin{abstract}
Saltwater intrusion is a transient process that affects the coastal aquifers quality and hydrodynamics. The transient behavior of the saltwater wedge (SW) and mixing zone (MZ) due to the changes of the inland freshwater head was investigated through experimental and numerical approaches using image processing technique and the numerical code SUTRA. To acquire data in the transient conditions, automated algorithms were designed and employed for both methods. Numerical simulations were extended to a reference problem of field scale for further study of the transient aspect of the saltwater intrusion phenomena. The results demonstrated that the behavior of SW area is significantly similar to the behavior of SW toe length in transient conditions. Also, in the advancing case, the SW height reaches the steady state condition much sooner than the SW toe length and the SW area, while in the receding case, all the three indicators are stabilized almost simultaneously. Furthermore, the results showed that the MZ expanded at early stages of the receding and after a while condensed again gradually until it finally reaches to its original state at the beginning of the advancing case. Although local velocity of brackish water toward sea boundary in the dilute region of the MZ is more than in the dense region, the flushing and mixing process causes to increase the MZ in the receding case. Sensitivity analyzes showed that the speed of SW advancing or receding does not affect the MZ thickness in a steady state condition.
\end{abstract}

\section{Introduction}

Coastal aquifers are main sources of freshwater supply in many coastal regions around the world. These resources are exposed to saltwater intrusion due to their proximity to seas. Saltwater intrusion is a natural phenomenon in which seawater would advance into the aquifer as a wedge since its density is higher than density of the fresh groundwater. The advancement of saltwater wedge (SW) is exacerbated by the groundwater head drop which could be a result of groundwater extraction $[1,2]$. The mixing zone (MZ), which is mainly the result of mechanical dispersion and molecular diffusion of dissolved matter, would be developed at the upper level of SW which is in contact with lower level of freshwater surface. MZ is an important feature of coastal aquifers across which the density and concentrations of saline water vary. Thereby, it has a significant contribution to saltwater circulation within the wedge and the transportation of salt to the freshwater outflow as well [3]. Field investigations show that thickness of mixing zone (TMZ) can extend from a few meters to several kilometers [e.g. 4, 5, 6]. Since mixing a small amount of saltwater with freshwater (even about $1 \%$ ) decreases the quality of water for drinking and other purposes, $\mathrm{MZ}$ is an important region in the coastal aquifers [7]. Better understanding of $\mathrm{MZ}$ and $\mathrm{SW}$ behavior in transient conditions and determining the effective parameters, play an important role in improving the management of coastal aquifers.

Many experimental studies have investigated the SW dynamic under the influence of various factors. In most cases, [e.g. 1, 2, 8-13] experimental data acquisition was based on visual observations. Determining the behavior of saltwater intrusion parameters (i.e. SW and $\mathrm{MZ}$ ) in transient conditions based on visual 
observations is time-consuming and involves errors of subjective [14]. Therefore, researchers have more focused on measuring the length of wedge toe $\left(L_{T}\right)$ as an indicator of the SW advancing at limited and specific times. Goswami and Clement [8] studied the effect of hydraulic gradient change on a headcontrolled system on behavior of SW. Chang and Clement [2] investigated the effect of groundwater discharge variation on a flux-controlled system on dynamics of SW. Although in both researches $L_{T}$ changes were visually measured at specific times, their study results were presented as benchmarks for verifying numerical models of variable-density flows. Chang and Clement [2] showed that timescale associated with wedge toe receding is less than its intrusion timescale. They cited the switch in flow field and creating unidirectional field of saltwater and freshwater toward the sea is as the reasons for this phenomenon.

A few laboratory experiments have been conducted to investigate the behavior of MZ in both steady and transient conditions. In laboratory models, due to their small scale, MZ has small thickness [3]. Therefore, the visual identification of $\mathrm{MZ}$ in laboratory models, especially in transient conditions, is hard or rather impossible [14]. Hence, in most laboratory studies, [e.g. 2, 8] MZ has been considered as a sharp interface. In one of few studies that focused on MZ determination in steady state, Abarca and Clement [7] through a colorimetric method, presented a proper visualization of the range of MZ in a physical model. The results of experiment showed that TMZ is thin in the wedge toe and is thickening along the interface toward the sea boundary in steady state condition. Lu et al. [15] investigated the effects of stratified aquifer heterogeneity on TMZ experimentally and numerically. According to their study, considering visual acquisition of data, TMZ changes were analyzed for steady state conditions. Robinson et al. [14] investigated the seawater intrusion process in a $38 \mathrm{~cm} \times 15 \mathrm{~cm} \times 1 \mathrm{~cm} \quad(\mathrm{~L} \times \mathrm{H} \times \mathrm{W})$ model using an automated image analysis technique. Their study was focused more on deriving calibration relationship which enabled to determine the dissolved solute concentration in each pixel of picture based on the light intensity. They mapped the variations of concentration for each image and used $50 \%$ concentration isoline to determine $\mathrm{L}_{\mathrm{T}}$ and $25 \%$ and $75 \%$ concentration isolines to determine the TMZ. Robinson et al. (2015) presented a new technique to identify the parameters associated with saltwater intrusion in small-scale laboratory models, but their investigation on behavior of SW and $\mathrm{MZ}$ was limited to measuring changes of $\mathrm{L}_{\mathrm{T}}$ and average thickness of the MZ between 0.2 to $0.8 \mathrm{~L}_{\mathrm{T}}$, respectively. Recently, Rezapoor et al. [16] using images analysis investigated effects decrease and increase freshwater head on behavior of wedge toe length and wedge height on the sea boundary in laboratory model. They show that in the case of the advancing wedge, the wedge height reaches the steady state condition much sooner than the wedge toe length, while in the case of the receding wedge, all the tow indicators are stabilized simultaneously

Numerical models can be an effective research tool to improve the understanding of the saltwater intrusion process. However, the numerical studies of saltwater intrusion in transient conditions have received less attention. In numerical modellings, similar to laboratory experiments, the process of data acquisition by visual observation and analyzing them in transient conditions, especially for MZ, is a hard work that faces many sources of errors. Therefore, most of previous numerical researches have been focused on steady state, including various forms of Henry problem [e.g. 17-21]. Considering dispersion effects in Henry problem and carrying out various numerical simulations, Abarca et al. [17] showed that both the longitudinal and transverse dispersivity, have the equal influence on TMZ. However, it is suggested that local transverse dispersion is the main reason for $\mathrm{MZ}$ development in steady states homogeneous aquifers $[19,22]$.

Reviewing the literature demonstrates that numerous valuable researches have been performed on the problem of saltwater intrusion. Despite this, there are still knowledge gaps in identifying and understanding this phenomenon in transient conditions [3]. This research was conducted both experimentally and numerically to clarify transient behavior of SW and MZ in head-controlled systems. In order to further study of the SW dynamics, in addition to examining $L_{T}$ changes that was used in most of the previous experimental and numerical studies, changes in two indicators of the saltwater wedge area $\left(S_{s w}\right)$ and the saltwater wedge height on the sea boundary $\left(h_{s w}\right)$ were investigated. To overcome the human constraints, the laboratory data were acquired using the image processing techniques and the numerical simulation results were automatically collected using the algorithmic implementation. The numerical modellings was developed on a large-scale (field-scale) system to further assessment and better interpretation of the observed phenomena in laboratory experiments.

\section{Materials and methods}

\subsection{Laboratory experiments}

\subsubsection{Laboratory equipment}

The experiments were conducted in a 2-D flow tank. The flow tank was constructed of transparent glass supported by aluminum profiles. The flow tank was divided into three distinct chambers consisting of a central chamber with internal dimensions $110 \times 65 \times 4$ (in $\mathrm{cm}$ ) and two side chambers of $5 \mathrm{~cm}$ length. The Side chambers were used to apply boundary conditions 
during the experiment. The central chamber was filled by $1-1.2 \mathrm{~mm}$ diameter glass beads $\left(2400 \mathrm{~kg} / \mathrm{m}^{3}\right)$. The Porosity of glass beads was measured as 0.37 by both volumetric and gravimetric methods. The netted Plexiglas screens were used to separate porous medium from the side chambers. The freshwater was supplied by a tap water at $24^{\circ} \mathrm{C}\left(997.30 \mathrm{~kg} / \mathrm{m}^{3}\right)$ and the saltwater was prepared by adding salt and red food dye to freshwater inside 60 liter barrels $\left(1021.25 \mathrm{~kg} / \mathrm{m}^{3}\right)$. Since it was planned to use the technique of image processing, the flow tank placed inside a darkroom (Figure 1). In order to adjust the light conditions during the experiment, a uniform light source including 8 fluorescent bulbs (20 watts) was provided. The bulbs were placed on a wooden panel in parallel and with equal distances. The interior walls of the darkroom were colored by mate black dye to prevent the reflection of light. The pictures were taken at specific intervals by a Nikon D7100 digital camera with resolution of $6000 \times 3368$ pixels in RAW format.

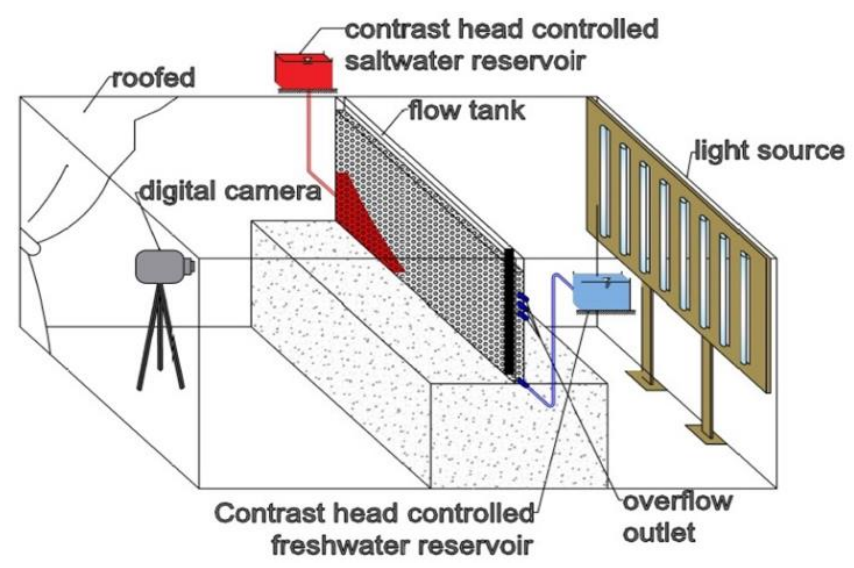

Figure 1. Schematic of the experimental set up.

\subsubsection{Experiment process}

Prior to the experiment, the glass beads were poured into the central chamber in $5 \mathrm{~cm}$ layers and were uniformly packed under fully saturated condition. Then freshwater was injected to the right chamber and passed through the porous media. The water heads in the right and left chambers were set to $48.8 \mathrm{~cm}$ and $46.88 \mathrm{~cm}$, respectively. After the flow reached the steady state, saltwater was injected to the left chamber from the bottom. Saltwater rapidly replaced the freshwater and its excess overflowed from the opening at $46.88 \mathrm{~cm}$ elevation. The process of saltwater intrusion began with formation of SW inside the porous media. As time passed, the SW intruded into aquifer until the system finally reached the steady state conditions (first stage of the experiment). After ensuring that the wedge was stabled, the freshwater head in the right chamber was decreased from $48.8 \mathrm{~cm}$ to $48 \mathrm{~cm}$ elevation. As it was expected, the SW started to advance and its advancement continued until it reached the new steady state condition (second stage or the wedge advancing stage, Figure 5 a). At the final stage of the experiment, the freshwater head was raised from elevation $48 \mathrm{~cm}$ to
$48.8 \mathrm{~cm}$. As a result, $\mathrm{SW}$ started to recede toward the sea boundary until it was finally stopped (third stage or the wedge receding stage, Figure $5 \mathrm{~b}$ ).

\subsubsection{Data acquisition and image processing}

$\mathrm{L}_{\mathrm{T}}$ was divided into four equal parts to analyze the TMZ along interface (Figure 2). The average vertical thickness of MZ was calculated in each part and applied to the next analyses.

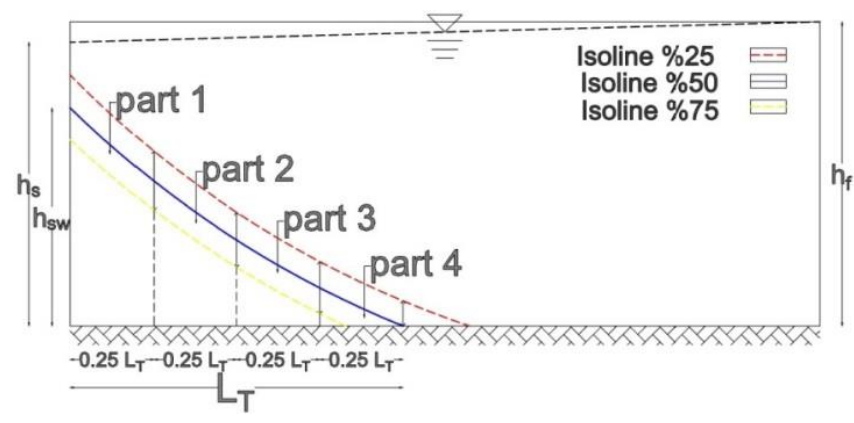

Figure 2. Reference diagram for measurement of TMZ along interface.

Prior to the experiments, light conditions inside the darkroom were set so that the camera's lens only received the light passing the central chamber. After a sensitivity test, the camera was settled in 1.5 meters from the flow tank and camera parameters were set to: focal length $35 \mathrm{~mm}$, shutter speed $0.33 \mathrm{sec}$, lens aperture $\mathrm{f} / 8$, ISO 320 , and white balance in florescent state. SW movement was captured in 60 -seconds intervals. In order to acquire the desired parameters in transient conditions, digital images were analyzed automatically. To this end, the proposed algorithm was implemented that included following stages:

\section{a) Pre-processing}

The purpose of this step was to remove areas outside the central chamber automatically so the first captured color image (Figure 3a) was converted into a gray image. Using a proper threshold we converted the gray image into binary image (Figure $3 \mathrm{~b}$ ). Using relevant regions of extracted binary image we aim to construct a mask to specify the region of interest during the experiment. For this purpose we employed closing operator from morphological operation to link close connected components. The component with the largest area in center of binary image considered as the region of interest. We use the bounding box of this region as a mask in the next steps to extract region of interest (Figure 3c) from all captured images during the experiment. 


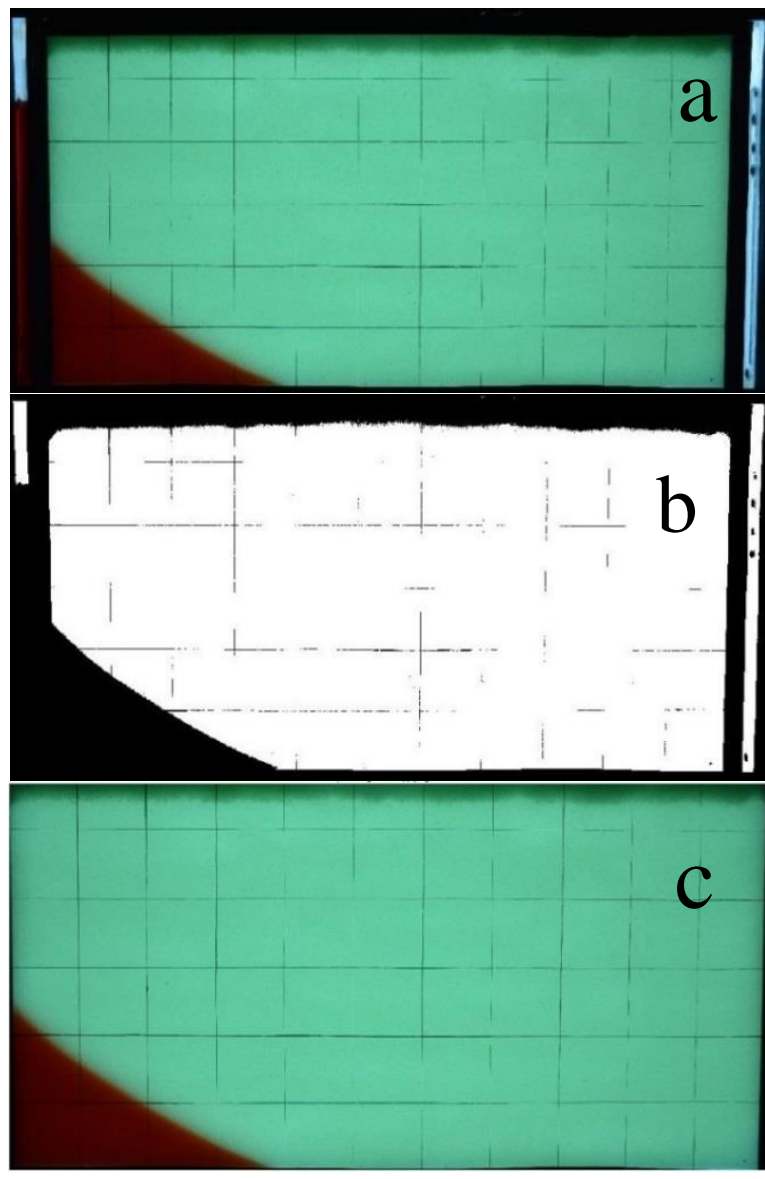

Figure 3. Pre-processing to remove areas outside the central chamber of the image. (a) Color image captured by camera, (b) Binary image, (c) Cropped image after masking.

\section{b) Data acquisition}

Considering that the HSI (hue, saturation, intensity) color space is more stable than RGB (red, green, blue) space, so we used HSI color space to identify SW in each captured image. In HSI model, channel H, S and I represent the color type (color wavelength), the saturation and intensity of pixels in image, respectively. Then a region of image with channel $\mathrm{H}$ close to red wavelength and also the concentration more than a specified threshold (the concentration of $50 \%$ saltwater and freshwater) were extracted as SW boundaries (Figure 4a). $S_{S w}$ in each pixel was computed by counting numbers of existing pixels in the wedge and multiplying them in the area of each pixel. It is worth noting that given the model dimensions (in $\mathrm{cm}$ ) and numbers of rows and columns of the corresponding digital image, area of each pixel of image was calculated as $1.46 \times 1.46 \mathrm{~cm}^{2}$. To determine $\mathrm{h}_{\mathrm{sw}}$, first horizontal projection of the wedge's image was obtained (Figure 4b). Then the point in which value of projection vector starts to increase from the zero was considered as $\mathrm{h}_{\mathrm{Sw}}$. In order to determine $\mathrm{L}_{\mathrm{T}}$, first vertical projection of the wedge's image was obtained (Figure 4c). Then we moved from the last component of the projection vector to the first component of this vector and the first non-zero component was indicated as $\mathrm{L}_{\mathrm{T}}$. For a better identifying of $\mathrm{MZ}$ along the interface, first its thickness was measured in five different points at $0.25 \mathrm{~L}_{\mathrm{T}}$ intervals in each image. Then, the average of each two consecutive values of these measures was considered as the mean thickness for the indicated quaternary regions along the interface. Our studies showed that the green channel in RGB color device is a good representation of color concentration in MZ. Therefore, for five mentioned points the profile of green channel was derived from the wedge image (Figure 4d). In MZ, the profile of green channel was increasing from a minimum value to a maximum value so based on preliminary experiments we noted that the thickness can be measured where the profile changes from 25 to $75 \%$ of its maximum value.

\section{c) Processing of the entire data}

All operations in steps $a$ and $b$ were conducted automatically. All captured images from the experiment and the associated data were plotted during the process.

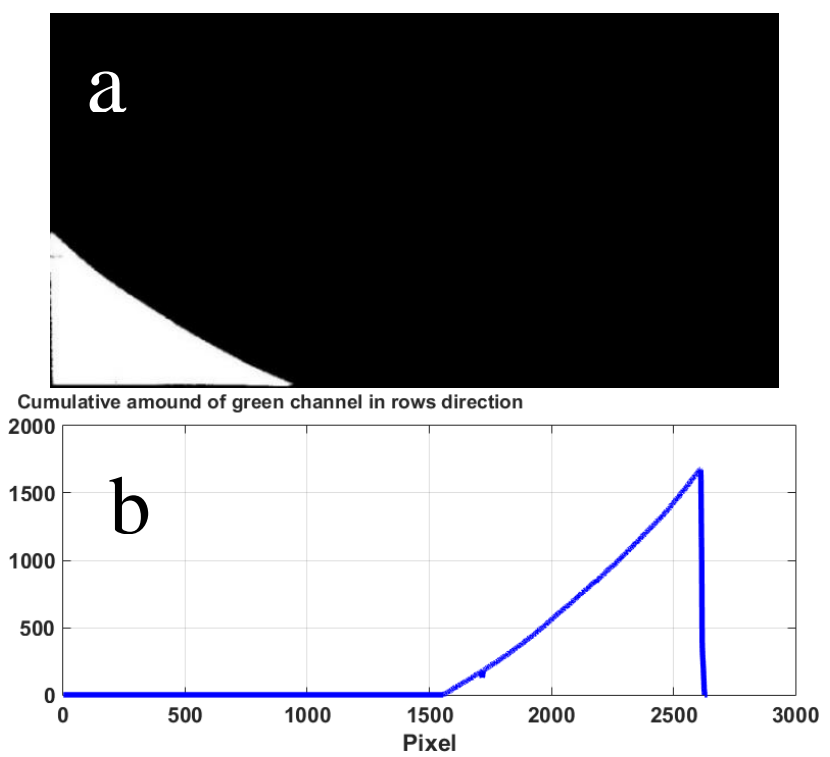

Cumulative amount of green channel in columns direction
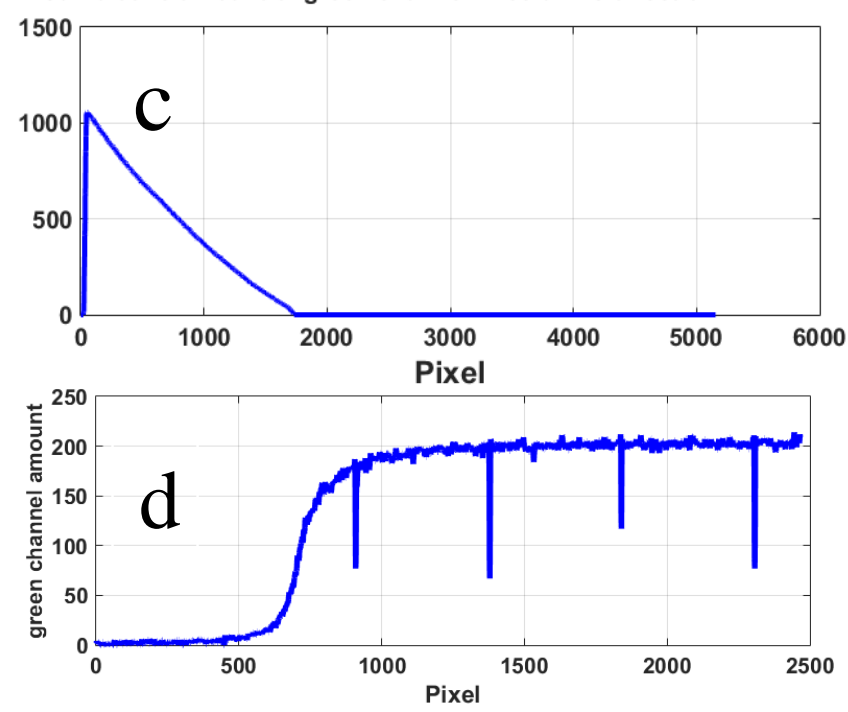

Figure 4. Data acquisition process. (a) Boundaries of SW in the HSI color space, (b) Horizontal projection of SW to determine $h_{s w}$, (c) Vertical projection of SW to determine $L_{T}$, (d) Variation of the green channel profile of the image to assess TMZ. 


\subsubsection{Determining the hydraulic conductivity}

In this study, two methods were used to compute hydraulic conductivity of porous medium. First, through a method similar to the one described in the literature [e.g. 8, 12, 13, 14, 23] hydraulic conductivity (K) was measured in porous medium under various hydraulic gradients, which returned the value of $\mathrm{K}$ in the range of 0.6 to $0.68 \mathrm{~cm} / \mathrm{s}$. In the second method image analysis was used. For this purpose, like the method explained in the previous section (experimental process) the freshwater flow passed through the porous medium from left to right under a given hydraulic gradient until it reached the steady state. This time the dyed freshwater (without salt) injected into the left chamber. The colored freshwater was rapidly replaced with the blank freshwater and overflow from a specific height. In this situation the dyed freshwater began to move across the cross section in the form of a blade in the direction of the main flow. From the moment the colored blade entered the porous medium until it has exit, the camera shot the colored water movement in 60 second intervals. The obtained images were analyzed. Then the time during which the dyed water passed through the porous medium was calculated for various elevations of $5,15,25$ and $35 \mathrm{~cm}$. Given the passing time and the porous media length, the actual velocity (pore velocity) was calculated for the abovementioned elevations. The actual velocity of porous media was obtained by averaging these velocities. The actual velocity was multiplied in the porosity of beads and Darcy velocity was obtained. Finally, the hydraulic conductivity of the porous media was computed using Darcy's law. This process was repeated for different hydraulic gradients. With this method, the hydraulic conductivity of the porous medium was estimated in the range of $0.63-0.67 \mathrm{~cm} / \mathrm{s}$. Eventually, The hydraulic conductivity in the porous media was considered as $0.65 \mathrm{~cm} / \mathrm{s}$ for the rest of the experiments.

\subsection{Numerical simulation}

The USGS code, saturated-unsaturated densitydependent flow and transport or SUTRA [24] was employed to simulate laboratory experiments and to perform the numerical simulations in the field scale. In order to simulate the laboratory data, a twodimensional rectangular domain with cross-section of $110 \mathrm{~cm} \times 46.88 \mathrm{~cm}$ was created. The saltwater and freshwater hydrostatic pressure was forced to the left and right boundaries, respectively. A no-flux condition was applied to the upper and lower boundaries of the model domain. The longitudinal and transverse dispersitivity values and the size of grid elements were determined through a trial and error process. Trial and error was begun by considering the proposed range of dispersivity in Abarca and Clement [7] and the spatial stability criterion of SUTRA [24]. The change in the longitudinal and transverse dispersitivity and diminution of the grid dimensions continued under two conditions. Firstly, the numerical results were in good agreement with the experimental data, and secondly, no change was observed in the results of the two consecutive simulations especially for MZ. Finally, a uniform grid with the dimensions of $\Delta X=4 \mathrm{~mm}$ and $\Delta \mathrm{Z}=3 \mathrm{~mm}$ which resulted in 42900 quadrilateral cells was used for modelling. The time step simulation was set to $\Delta t=1 \mathrm{sec}$. A summary of the used parameters in SUTRA model for simulation of the laboratory experiments are listed in Table 1. In addition to simulate laboratory experiments, field scale simulations were performed to confirm that the laboratory observations occur on a larger aquifer and to better interpretation experimental results. A twodimensional Hypothetical aquifer was considered for numerical modeling in field scale, called base- case. Geometric characteristics and the hydrogeological parameters considered in the base-case followed those of Lu and Werner [25] as seen in Table 1. The aquifer was isotropic and homogenous with $\mathrm{L}=1000 \mathrm{~m}$ length and $\mathrm{h}=30 \mathrm{~m}$ thickness. The sea was on the left side of the aquifer and its level was at $30 \mathrm{~m}$ of the aquifer bed. In the base-case, freshwater head on the right boundary of the aquifer (inland boundary) changes from $32 \mathrm{~m}$ to $31 \mathrm{~m}$. For proper simulation of $\mathrm{MZ}$ similar to $\mathrm{Lu}$ and Werner's work [25] the model domain was segmented to a small grid with the dimensions $\Delta x=1 \mathrm{~m}$ and $\Delta z=0.5 \mathrm{~m}$, resulting in 60000 quadrilateral cells. The time step was set to $\Delta t=1$ day. Similar to the laboratory case, the boundary conditions of the model domain was applied by considering the dimensions of the base-case. In order to identify SW and MZ behavior in transient conditions, the SW indicators and thickness of four MZ part have to be collected and analyzed in consecutive intervals. Due to the existence of multiple parameters and the long-time to reach to steady state, collecting data directly from SUTRA and then analyzing them were not practical. Therefore, to ease the operation and to improve the accuracy and speed, it was necessary to collect and analyze the data in an automatic process. After completing the simulation, the salinity concentration data were exported to CSV file in all the time steps. The concentration matrix was imported to another computer code for further illustration. An algorithm with the following steps was implemented in this code: a) Concentration contours were plotted with high accuracy in each time step, b) All considered parameters, including $L_{T}, S_{s w}, h_{s w}$ and the average thickness of various parts of MZ were measured, c) a and b steps are automatically implemented during wedge's intrusion and its recessing and changes in each parameter were plotted in relation whit time. 
Table 1. Summary of parameters used in Sutra

\begin{tabular}{|c|c|c|c|}
\hline \multirow[b]{2}{*}{ Parameter } & \multirow[b]{2}{*}{ Symbol } & \multicolumn{2}{|c|}{ Value } \\
\hline & & $\begin{array}{l}\text { Laboratory- } \\
\text { Scale }\end{array}$ & $\begin{array}{l}\text { Field-Scale } \\
\text { (Base-Case) }\end{array}$ \\
\hline Aquifer length (m) & $\mathrm{L}$ & 1.1 & 1000 \\
\hline $\begin{array}{l}\text { Aquifer thickness } \\
\text { (m) }\end{array}$ & B & 0.4688 & 30 \\
\hline Permeability $\left(\mathrm{m}^{2}\right)$ & $\mathrm{k}$ & $6.5 \mathrm{E}-10$ & $1.18 \mathrm{E}-11$ \\
\hline Effect porosity (-) & $\mathrm{n}$ & 0.37 & 0.3 \\
\hline $\begin{array}{l}\text { Freshwater density } \\
\left(\mathrm{kg} / \mathrm{m}^{3}\right)\end{array}$ & $\rho_{f}$ & 997.3 & 1000 \\
\hline $\begin{array}{l}\text { Saltwater density } \\
\left(\mathrm{kg} / \mathrm{m}^{3}\right)\end{array}$ & $\rho_{s}$ & 1021.25 & 1025 \\
\hline $\begin{array}{l}\text { Longitudinal } \\
\text { dispersivity (m) }\end{array}$ & $\alpha_{L}$ & 0.001 & 1 \\
\hline $\begin{array}{l}\text { Transverse } \\
\text { dispersivity (m) }\end{array}$ & $\alpha_{T}$ & 0.000075 & 0.1 \\
\hline $\begin{array}{l}\text { Solute mass fraction } \\
\text { of saltwater (-) }\end{array}$ & $C_{s}$ & 0.035 & 0.035 \\
\hline $\begin{array}{l}\text { Specific storage } \\
(1 / \mathrm{m})\end{array}$ & $S_{S}$ & 0.008 & 0.008 \\
\hline $\begin{array}{l}\text { Gravitational } \\
\text { acceleration }\left(\mathrm{m}^{2} / \mathrm{s}\right)\end{array}$ & $\mathrm{g}$ & 9.81 & 9.81 \\
\hline
\end{tabular}

\section{Results and discussion}

\subsection{SW behavior}

\subsubsection{Experiment results in the laboratory scale}

In the steady state conditions of the first stage, $L_{T}, S_{S w}$ and $h_{s w}$ were measured $38.24 \mathrm{~cm}, 378.33 \mathrm{~cm}^{2}$ and $24.52 \mathrm{~cm}$, respectively. Figure 5 shows the digital images of advancing SW and its receding inside the central chamber. In these images, the numerical simulations results for $50 \%$ saltwater concentration isoline are shown with the white circles. Generally, there is an agreement between the experimental data and the numerical simulations results especially in the wedge toe. With regards to the interface, the numerical simulation results show more curvature than the experimental data, especially near the sea boundary. Robinson et al [14] reported the partial heterogeneities, which is due to small changes in the beads diameter, as porous media, as the reason for more linear interface in physical models than in the numerical models. Reduction of the freshwater head at the beginning of the advancing stage decreases interactional forces applied to SW by freshwater. As a result, the equilibrium in the aquifer system is disrupted and SW intrudes into the aquifer until it reaches the new equilibrium state (Figure 5a). In contrast, by increasing the freshwater head, the interactional forces of freshwater against SW increase and the wedge recedes toward the sea (Figure 5b).
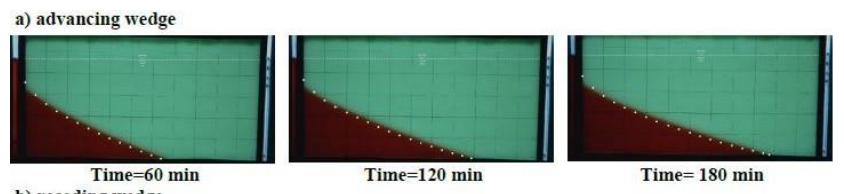

b) receding wedg
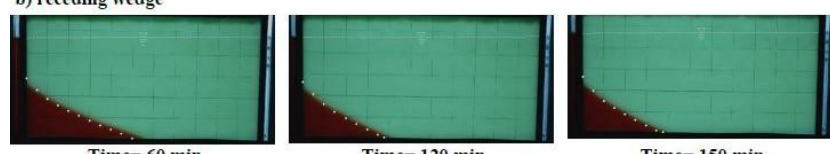

Figure 5. SW movement in the physical model. White circles show the numerical results based on concentration of $50 \%$ saltwater. (a) Advancing SW due to instantaneous decrease in freshwater head from 48.8 to $48 \mathrm{~cm}$. (b) Receding $S W$ due to instantaneous increase in freshwater head from 48 to $48.8 \mathrm{~cm}$.

Figure 6 shows the transient behavior of the SW indicators based on the numerical and experimental results. Both numerical and experimental results were automatically acquired based on $50 \%$ saltwater concentration isoline. A good correlation between experimental and numerical results in transient conditions for $L_{T}$ and $S_{S w}$ is concluded from Figures $6 \mathrm{a}, 6 \mathrm{~b}$, respectively. The trend of changes in $h_{s w}$ is similar in both numerical and experimental modelling under transient condition; however a small difference between numerical and experimental results was detected (Figure $6 \mathrm{c}$ ) as mentioned earlier in this text. A glance at the Figure 6a shows that the $L_{T}$ indicator reaches a steady state in the receding case in a shorter period of time than in the intruding case. The timescale for $L_{T}$ receding is almost 130 minutes, while it is about 175 minutes for intruding. This phenomenon was first reported by Chang and Clement [2] and then it was observed in works of Lu and Werner [25] and Robinson et al. $[14,26]$. A closer look at the graphs in Figure 6 shows that over time, the trend of $S_{S W}$ changes is similar to the trend of $L_{T}$ changes, while these state for $h_{s w}$ is different from two other indicators (Figure 6c). To clarify this issue, changes of all three indicators over time are plotted in a single figure. From Figure 7 forward, in which the transient behavior of $L_{T}, S_{s w}$ and $h_{s w}$ were compared, the minimum and maximum values of the vertical axes of graphs were adjusted in a way that the initial and the end points of the graphs in both stages (advancing and receding) are coincide. This allows the possibility of comparing the relative rate of change of each indicator at any stage (i.e., the rate of change of each indicator in relation with the total change of that indicator at each stage). In addition, it shows the amount of each indicator in transitional conditions according to its own axis. Figure 7 shows that most of the time, relative rate of change of $S_{S w}$ equals to relative rate of change of $L_{T}$ (i.e. the slopes of a tangent line of both curves are almost equal at any times). 


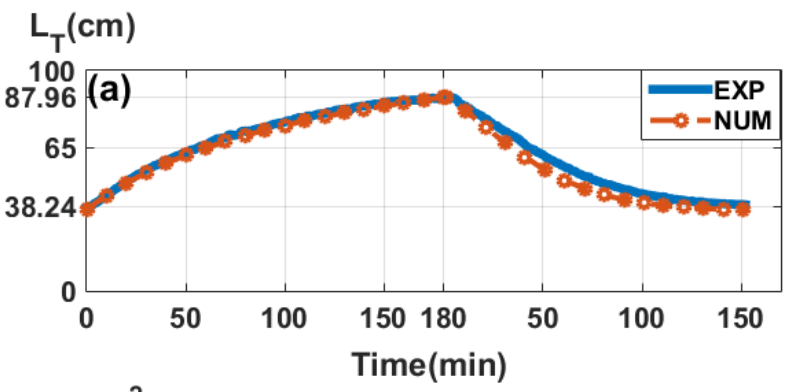

$\mathrm{S}_{\mathrm{sw}}\left(\mathrm{cm}^{2}\right)$
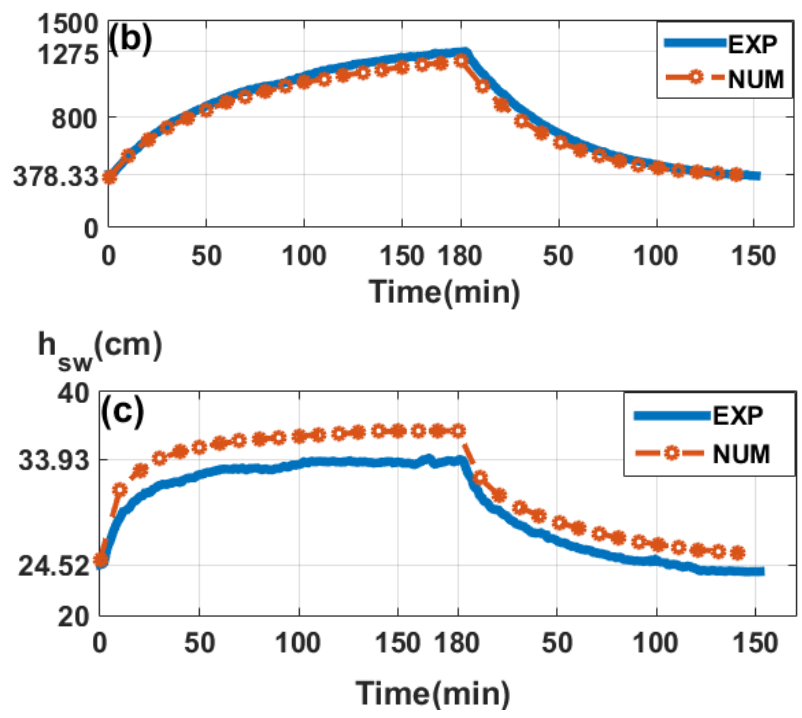

Figure 6. Transient behavior of $S W$ (a) $L_{T}$, (b) $S_{s w}$, and (c) $h_{s w}$ in the physical and numerical experiments. Advancing during $0<t<180 \mathrm{~min}$ and receding $\mathrm{t}>180 \mathrm{~min}$

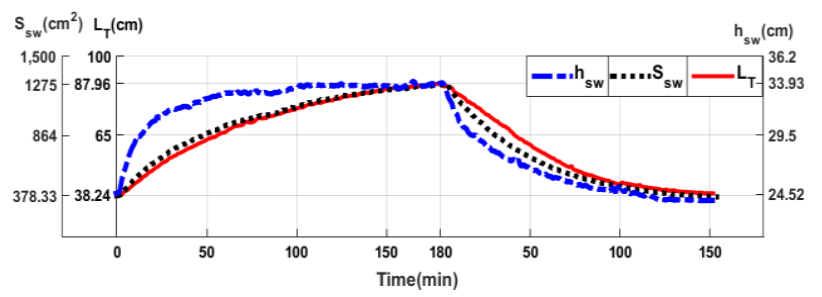

Figure 7. Comparative graph for transient behavior of $L_{T}, S_{s w}$, and $h_{\text {sw }}$ in the physical model

The laboratory results show that $\mathrm{L}_{\mathrm{T}}$ indicator can be a suitable representative to show the intruded saltwater volume to coastal aquifers not only in the steady state but also in the transient state. Another notable point is the transient behavior of $h_{s w}$. In the case of advancing $\mathrm{SW}, h_{s w}$ changes at a much higher relative rate than $L_{T}$ and $S_{S W}$ in a way it reaches the steady state before $L_{T}$ and $S_{S w}$, while all three indicators stabilize almost simultaneously in the SW receding case. Due to the density difference between the freshwater and saltwater, the interface advancement in steady conditions increases by increasing depth. As the steady interface advancement increases, the relative rate of its advancement decreases. Therefore, the height of wedge heel $\left(h_{s w}\right)$ has the highest relative movement in the interface that reaches the steady state before other points. For receding SW, since $h_{s w}$ is in the vicinity of the sea boundary, it decreases more rapidly than $\mathrm{L}_{\mathrm{T}}$ at the beginning of the receding stage. But after a while, the freshwater enters the $\mathrm{MZ}$ and starts to flush it. The brackish waters move along the interface toward the outflow at the sea boundary. Passing of brackish waters from the sea boundary causes the decrease in rate of drop of $h_{s w}$. The flushing and movement of the brackish waters toward the outflow continue while the toe approaches the steady state. As a result, all mentioned above three indicators of SW achieve the steady state almost simultaneously.

\subsubsection{Numerical results in field scale}

Figure 8 shows the changes of $L_{T}, S_{s w}$ and $h_{s w}$ over time for the base-case aquifer. It indicates that the transient behavior of SW in the field-scale model (basecase) is similar to our experimental-scale model. In general: a) relative rate of changes of $L_{T}$ and $S_{S w}$ are rather equivalent at the same times, in other words, these indicators show similar behavior in transient conditions, b) In the advancing case, $\mathrm{h}_{\mathrm{sw}}$ reaches the steady state much sooner than the two other indicators because of its higher relative rate of change. Note that according to Watson et al. [27] and $\mathrm{Lu}$ and Werner [25] studies, if timescales of each indicator is considered as $95 \%$ of the total duration of the indicator displacement, $\mathrm{h}_{\text {sw }}$ reaches the steady- state condition at 35 year while $L_{T}$ and $S_{S w}$ reach the steady-state condition at 65 year. c) In the receding case, all the three indicators stabilize almost simultaneously.

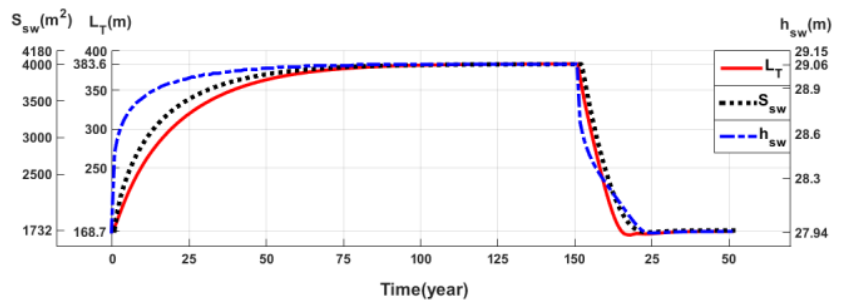

Figure 8. Comparative graph for transient behavior of $L_{T}, S_{s w}$, and $h_{s w}$ in the field scale (Base-Case)

\subsubsection{Sensitivity of $\mathrm{SW}$ behavior to the rate of inland head change}

In order to examine the sensitivity of the SW indicators to the rate of inland head change, In addition to the instantaneous case (base-case), three scenarios for change in the rate of inland head change were considered: $0.5 \mathrm{~mm} /$ day, $0.1 \mathrm{~mm} /$ day and $10 \mathrm{~mm} /$ year. The simulation results suggested that firstly, the previous findings of this research are valid for all scenarios. Secondly, the less the rate of inland head change, the more the agreement between relative rates of change of $S_{\mathrm{Sw}}$ to the relative rate of change of $L_{T}$ exists. For instance, Figure 9 demonstrates the trend of change in $L_{T}, \mathrm{~S}_{\mathrm{sw}}$ and $\mathrm{h}_{\mathrm{sw}}$ over time for the case of $10 \mathrm{~mm} /$ year. 


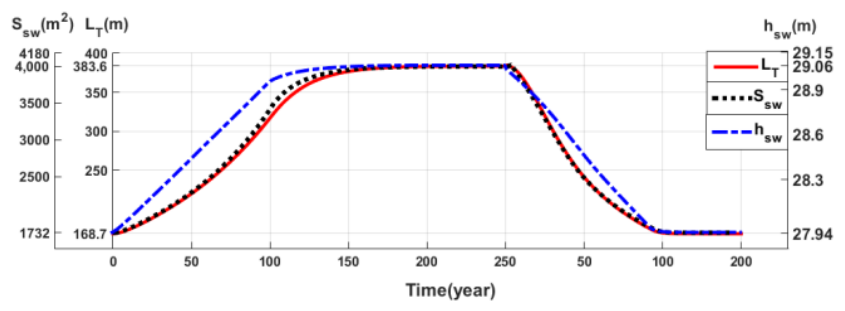

Figure 9. Comparative graph for transient behavior of $L_{T}, S_{s w}$, and $h_{s w}$ in the field scale (the rate of inland head change $10 \mathrm{~mm} /$ year).

\subsubsection{Sensitivity of $\mathrm{SW}$ behavior to dispersivity}

Figure 10 shows the transient behavior of $L_{T}$ for various combinations of longitudinal and transverse dispersivities. As it is expected, increasing any of dispersitivities decreases the wedge toe length as well as the time of reaching the steady state. However, the transverse dispersitivity is more effective on $L_{T}$ than longitudinal dispersitivity. It is worth noting that the behavior of $\mathrm{S}_{\mathrm{Sw}}$ is not shown due to its similarity to the behavior of $L_{T}$.

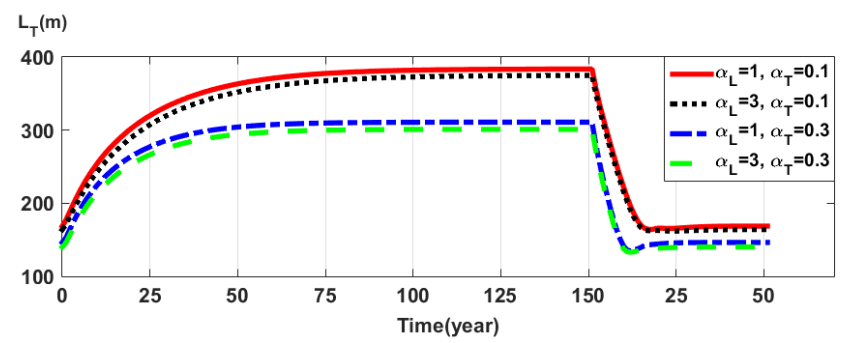

Figure 10. Sensitivity of $L_{T}$ to different combination of dispersivities.

\subsection{MZ behavior}

\subsubsection{Experiment results in laboratory scale}

The technique of image processing makes it possible to identify TMZ along the interface in the transient conditions. Figure 11 represents TMZ changes during the saltwater intrusion and retreat in physical model in four distinct parts. The numerical results are plotted in Figure 12. A comparison between Figure 11 and Figure 12 demonstrates that there is a small difference between the experimental and numerical results in some of the distinct parts and in some intervals. However, the trend of changes in TMZ is the same in both models. This indicates that SUTRA has an acceptable ability to simulate $\mathrm{MZ}$ in transient conditions. The results of both experimental and numerical modeling show that MZ is thin in Part 4 (top of the wedge toe) and becomes progressively thicker along the interface toward the sea boundary. The results also show that TMZ increases in all parts at the initial time periods of the receding stage. By approaching a maximum value, it decreases gradually until it finally reaches its initial value at the beginning of the intrusion stage. This phenomenon was also observed in Robinson et al. [14] laboratory experiments. By offering a general reason, they expressed that the occurrence of this phenomenon is associated with the change in the flow field at receding case and creating new highly disturbed flow field. In this research, this phenomenon was investigated using numerical modelling in the field-scale to avoid scale effect in laboratory tests as well as acquiring velocity data to examine the flow filed condition.

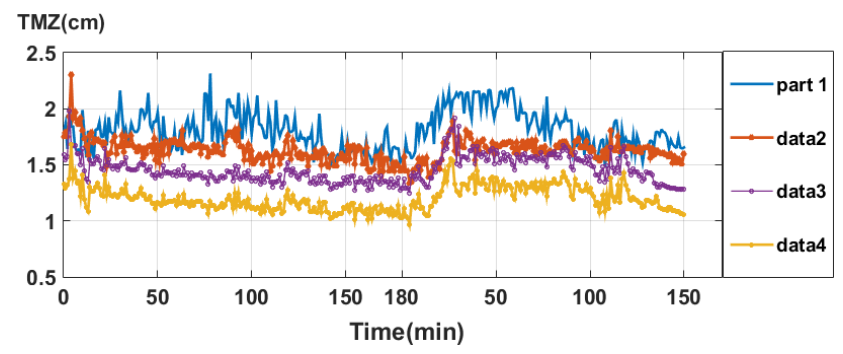

Figure 11. TMZ along the interface under transient condition in physical model

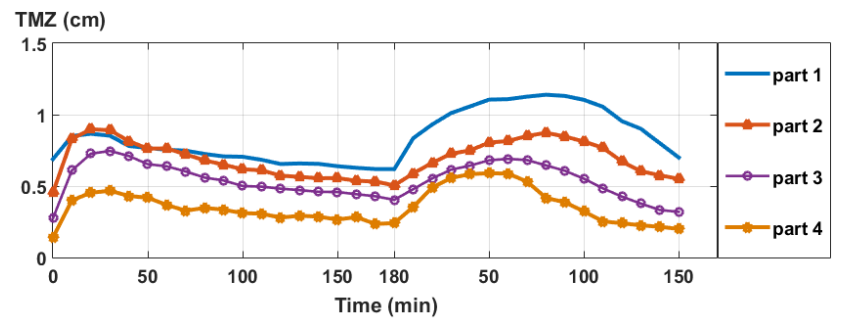

Figure 12. Numerical simulation results of TMZ along the interface under transient condition at laboratory scale

\subsubsection{Numerical results in the field-scale}

Figure 13 shows TMZ changes in the base-case under transient conditions. As seen, the MZ temporal change in field scale is similar to change in the laboratory scale. TMZ in both scales increases at the beginning of the receding stage and then decreases gradually until it finally reaches its initial value at the beginning of the intrusion stage. To analyze this phenomenon, $\mathrm{MZ}$ is supposed to be divided into several distinct layers between $10-25 \%, 25-50 \%, 50-75 \%$ and $75-90 \%$ saltwater concentration isolines. Obviously, due to the existence of the concentration gradient across MZ, each layer can be divided into smaller layers. It is known that the rise in inland freshwater head at the beginning of the receding case increases the freshwater flux into the aquifer and changes the movement direction of SW from the land toward the sea. Due to the lower velocity of SW in comparison to the freshwater flux, the freshwater near the SW would change direction along interface toward the outflow zone (at top of the SW on the sea boundary) and amount of the freshwater enter the MZ. By entering into the first hypothetical layer of MZ (between $10 \%$ to $25 \%$ isoline), freshwater mixed with brackish water. This leads to dilution of the layer while brackish water starts to flush its dense region (e.g. location $25 \%$ isoline). The flushing process transports some of salts solute along the interface toward the outflow zone, Thereby layer becomes diluted and 25\% contour closer to sea boundary (Figure 14). Although the fluid velocity toward the sea 
boundary in location of the dilute contour (10\% isoline) is greater than in location of the dense contour $(25 \%$ isoline), the conjoint effect of flushing, mixing and the local velocity cause the dense contour lengthen its distance from the dilute contour and the thickness of the layer extend. This process can be repeatedly occur until the brackish flows of the first layer enter the low adjacent layer and it can be repeated in the lower layers in the same way as well. After some period of time, the velocity difference between the freshwater flows and interface gradually decreases, resulting in the flushing and mixing processes gradually reduces. The dilute contours approach the dense contours in each layer and MZ inchmeal condenses. Finally, the wedge reaches the steady state and the thickness of MZ becomes equal to its thickness at the beginning of the intrusion stage. Figure 15 shows the changes of horizontal distance in the selected layers on the bottom of the aquifer (as a sample of the point whole each isoline along the interface) in transient conditions. It is observed that when the receding process begins, different layers of the MZ do not show an identical behavior across the zone. In more diluted layers, e.g. $10 \%$ to $75 \%$ isolines, the distances between the isolines expands while for $75 \%$ to $90 \%$, the isolines would remain almost the same as advancing case. This can be explained by the fact that the flushing and mixing processes continue as long as the brackish water flows can enter the lower layers. Since the velocity of the brackish flows gradually decreases as it gets closer to the sea boundary, brackish flows cannot enter into above-mentioned layer.

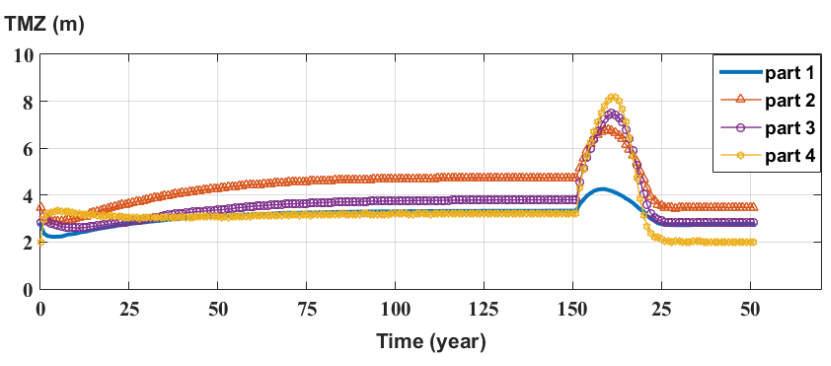

Figure 13. Field scale numerical simulation of TMZ along the interface under transient condition (Base-Case).

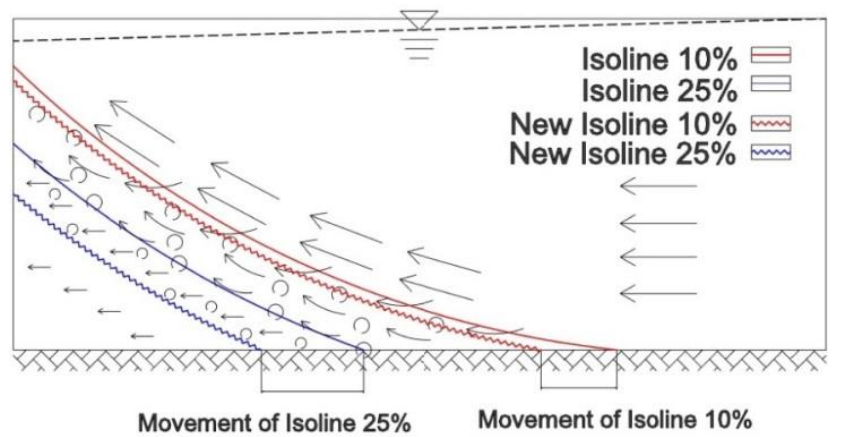

Figure 14. Schematic of expanding MZ. Due to flushing process, Dense Contour moves toward the sea boundary faster than dilute contour

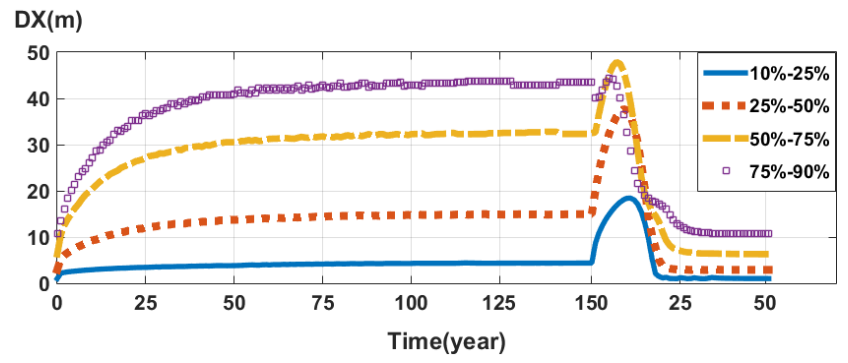

Figure 15. Changes of horizontal distance of selected contours on the aquifer bed under transitional condition (Base-Case).

\subsubsection{Sensitivity of $M Z$ behavior to the rate of the inland head change}

Figure 16 illustrates the transient behavior of the part 2 of $\mathrm{MZ}$ as a sample of the whole $\mathrm{MZ}$ under various scenarios for decreasing and increasing inland head. As it can be seen, the rate of inland head change has no influence on the TMZ in both intruding and receding cases under the steady state conditions. Figure 16 also shows that whatever the rate of rise of freshwater head decreases, the increase of the MZ thickness that occurs during the receding of SW, decreases. Since the freshwater flux into the aquifer declines, the possibility of entrance of brackish water to the various layers of $\mathrm{MZ}$ decreases and this causes the influence of flushing process in the layers to decrease.

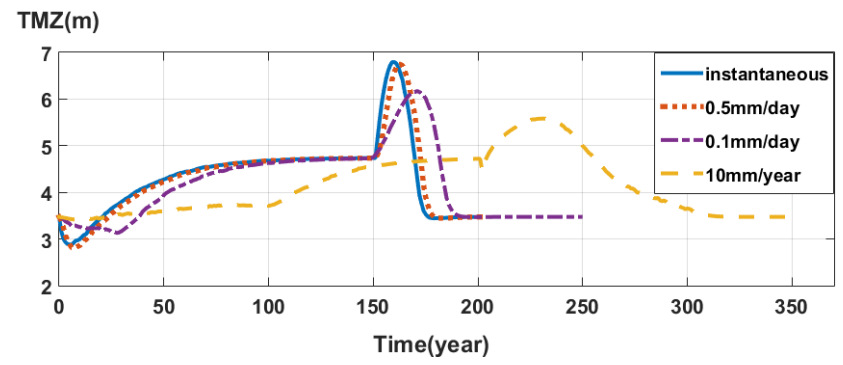

Figure 16. Sensitivity of TMZ to the rate of changes in land (Part 2).

\subsubsection{Sensitivity of MZ behavior to dispersivities}

Figures 17 show the results of numerical simulations for different combinations of dispersivities for the second parts of the MZ. By comparing the graphs of cases $\alpha_{L}=1, \alpha_{T}=0.1$ with $\alpha_{L}=3, \alpha_{T}=0.1$ or comparing cases $\alpha_{L}=1, \alpha_{T}=0.3$ with $\alpha_{L}=3, \alpha_{T}=0.3$ it is concluded that longitudinal dispersivity at early years of SW intrusion, in which saltwater intrusion is fast, has more influence on TMZ than the years leading to steady state in which saltwater intrusion is slow. On the other hand, comparing graphs of cases $\alpha_{L}=1, \alpha_{T}=0.1$ with $\alpha_{L}=1$, $\alpha_{T}=0.3$ or comparing graphs of cases $\alpha_{L}=3, \alpha_{T}=0.1$ with $\alpha_{L}=3, \quad \alpha_{T}=0.3$ indicates the transverse dispersitivities in slow saltwater intrusion has likely more impact on the MZ thickness than fast saltwater intrusion. However further investigation is required. 


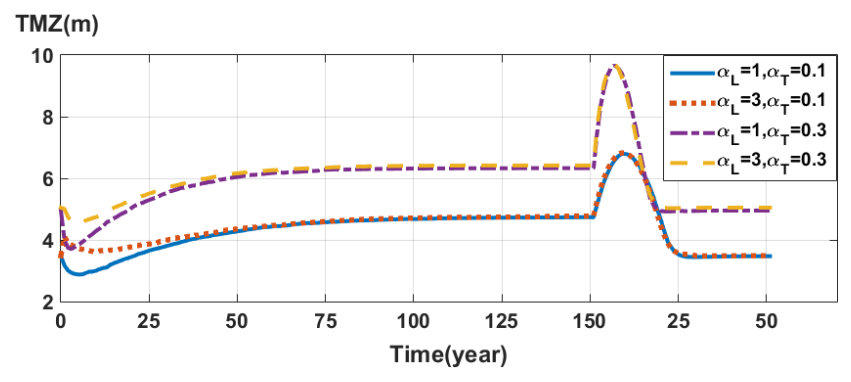

Figure 17. Sensitivity of TMZ to different combination of dispersivities (Part 2).

\section{Conclusion}

The dynamics of SW and MZ due to the change in the inland freshwater head were investigated experimentally and numerically. For both approaches, automatic data acquisition was employed and the results then were analyzed through the proposed algorithms. For SW, the trend of changes in the indicators namely $L_{T}, S_{s w}$ and $h_{s w}$ was evaluated. To extend the validity of the findings, the results from a laboratory scale were examined by a field scale sample through numerical experiment. The main findings of the research are:

1) The relative rate of change of $S_{S w}$ is equal to the relative rate of change $L_{T}$.

2 ) In the case of advancing $S W, h_{s w}$ reaches the steady state condition before $L_{T}$ and $S_{S W}$ since it changes at a much higher relative rate than $L_{T}$ and $S_{S W}$ state.

3) At the beginning of stage of the SW receding, the $h_{s w}$ moves more quickly than the $L_{T}$. But after a while, the flows of freshwater enter the MZ and start to flush it. The brackish flows move to the outflow on the sea boundary that results in reducing the velocity of the $h_{s w}$ descend. The operation of flushing and movement of the brackish water toward the outflow continue until the $L_{T}$ stops; as a result, $h_{s w}$ and $L_{T}$ reach the steady state almost simultaneously.

4). Although the velocity of brackish flows toward the sea boundary in the dilute layers of the $\mathrm{MZ}$ is more than its dense layers at early case of the SW receding, the flushing process transports some of salts of the MZ dense layers toward outflowing at sea boundary. This process along with mixing leads to dilution of the MZ dense layers and TMZ increases. As the wedge approaches the steady state, the processes of flushing diminishes, the dilution layers of MZ become close to its the dense layers, and the MZ shrinks again. Finally, the wedge reaches the steady state and the thickness of $\mathrm{MZ}$ becomes equal to its thickness at the beginning of the intrusion stage.

5) The rate of change of the freshwater head does not affect the steady magnitude of the TMZ, neither in the advancing nor in the receding case. However, the decrease in the rate of rise of the freshwater head limits the increase in the TMZ occurring at the beginning of the receding stage.
The findings of this study can provide a proper view of the SW and MZ movement in transient conditions for coastal groundwater resources management. On the other hand, the experimental and numerical results of this study have been obtained for a two-dimensional homogeneous aquifer with ideal boundary conditions. Applied field conditions, field scale heterogeneity, seawater and groundwater level fluctuations and the three-dimensional form of the aquifer are some of the issues whose effects on SW and MZ's transient behavior should also be considered. Finally, the experimental and numerical investigations can be performed for an aquifer with flux-controlled system and the SW and MZ dynamics for both systems can be compared

\section{References:}

1-Kuan, W. K., Jin, G., Xin, P., Robinson, C., Gibbes, B. Li, L., (2012), Tidal influence on seawater intrusion in unconfined coastal aquifers. Water Resources Research, 48:W02502 https://doi.org/10.1029/2011WR010678

2- Chang, S. W., Clement, T. P., (2012), Experimental and Numerical Investigation of Saltwater Intrusion Dynamics in Flux-Controlled Groundwater Systems. 48(March), 1-10.

3-Werner, A.D. et al. (2013), Seawater Intrusion Processes, Investigation and Management: Recent Advances and Future Challenges, Advances in Water Resources, 51: 3-26. http://dx.doi.org/10.1016/j.advwatres.2012.03.004.

4- Barlow, P.M., (2003), Ground Water in FreshwaterSaltwater Environments of the Atlantic coast. U.S. Geological Survey Circular 1262.

5- Price, R.M., Top, Z., Happell, J. D., Swart, P.K., (2003), Use of tritium and helium to define groundwater flow conditions in Everglades National Park

6- Cherry, G. S., (2006), U.S. Geological Survey Georgia Water Science Center and City of BrunswickGlynn County Cooperative Water Program - Summary of Activities, July 2005 through June 2006, U.S.G.S. Open-File Report 2006-1368.

7- Abarca, E., Clement, T. P., (2009), A Novel Approach for characterizing the mixing zone of a Saltwater Wedge, Geophysical Research Letters. 36(6). $1-5$.

8- Goswami, R. R., Clement, T. P, (2007), LaboratoryScale Investigation of Saltwater Intrusion Dynamics, Water Resources Research. 43(4), 1-11.

9- Luyun, R, Momii, K. and Nakagawa, K., (2009), Laboratory-Scale Saltwater Behavior due to Subsurface Cutoff Wall. Journal of Hydrology. 377(34), 227-36.

http://dx.doi.org/10.1016/j.jhydrol.2009.08.019. 
10-Luyun, R., Momii, K., Nakagawa, K., (2011), Effects of recharge wells and flow barriers on seawater intrusion. Ground Water 49, 239e249

11- Mehdizadeh, S., Werner, A. D., Vafaie, F., and Badaruddin, S., (2014), Vertical Leakage in SharpInterface Seawater Intrusion Models of Layered Coastal Aquifers, Journal of Hydrology. 519, $1097-$ 1107. http://dx.doi.org/10.1016/j.jhydrol.2014.08.027. 12- Oz, I., Shalev, E., Yechieli, Y., Gavrieli, I., Gvirtzman, H., (2014), Flow Dynamics and Salt Transport in a Coastal Aquifer Driven by a Stratified Saltwater Body: Lab Experiment and Numerical Modeling, Journal of Hydrology 511: 665-74. http://dx.doi.org/10.1016/j.jhydrol.2014.02.020.

13- Oz, I., Shalev, E., Yechieli, Y., and Gvirtzman, H. (2015), Saltwater Circulation Patterns within the Freshwater-Saltwater Interface in Coastal Aquifers: Laboratory Experiments and Numerical Modeling. Journal of Hydrology 530: 734-41. http://dx.doi.org/10.1016/j.jhydrol.2015.10.033.

14- Robinson, G., Hamill, G. A., Ahmed A. A., (2015), Automated Image Analysis for Experimental Investigations of Salt Water Intrusion in Coastal Aquifers. Journal of Hydrology. 530: 350-60. http://dx.doi.org/10.1016/j.jhydrol.2015.09.046.

15- Lu, Chunhui, Yiming Chen, Chang Zhang, and Jian Luo, (2013), Steady-State Freshwater-Seawater Mixing Zone in Stratified Coastal Aquifers. Journal of Hydrology, 505: 24-34. http://dx.doi.org/10.1016/j.jhydrol.2013.09.017.

16- Rezapoor, A. A., Saghravani.S. F., Ahmadifard, A. R., (2018), (in Farsi), Study of saltwater intrusion phenomenon in the coastal aquifers under transient condition using image processing and numerical modelling. Journal of hydraulics. 13(2), 69-82.

17- Abarca, E., Carrera, J., Sánchez-Vila, X., Dentz, M., (2007), Anisotropic Dispersive Henry Problem. Advances in Water Resources. 30(4). 913-926.

18- Simpson, M. J., and Clement, T. P., (2004), Improving the Worthiness of the Henry Problem as a Benchmark for Density-Dependent Groundwater Flow Models. Water Resources Research. 40(1), 1-12. http://doi.wiley.com/10.1029/2003WR002199.
19- Held, R., Attinger, S., Kinzelbach, W., (2005), Homogenization and Effective Parameters for the Henry Problem in Heterogeneous Formations. Water Resources Research. 41(11), 1-14.

20- Sanz, E., Voss, C. I., (2006), Inverse Modeling for Seawater Intrusion in Coastal Aquifers: Insights about Parameter Sensitivities, Variances, Correlations and Estimation Procedures Derived from the Henry Problem. Advances in Water Resources. 29(3): 43957.

21- Sebben, M.L., Werner, A.D., (2015), Seawater intrusion in fractured coastal aquifers: a Preliminary numerical investigation using a fractured Henry problem. Adv. Water Resour. 85, 93-108. http://dx.doi.org/10.1016/j.advwatres.2015.09.013.

. Res., 28(8): 2123-2134.

22- Paster, A., Dagan, G., (2007), Mixing at the Interface between Two Fluids in Porous Media: A Boundary-Layer Solution. Journal of Fluid Mechanics 584: 455. http://www.journals.cambridge.org/abstract_S002211 2007006532.

23- Oostrom, M., Hayworth, J. S., Dane, J. H., Guven, O., (1992), Behavior of dense aqueous phase leachate plumes in homogenous porous media, Water Resour 24-Voss, C., Provost, A., (2010), SUTRA—a Model for Saturated-unsaturated, Variabledensity

Ground-water Flow with Solute or Energy Transport, U.S Geological Survey Water-Resources Investigations Report, 02-4231.

25- Lu, C., Werner, A. D., (2013), Advances in Water Resources Timescales of Seawater Intrusion and Retreat, Advances in Water Resources 59, 39-51. http://dx.doi.org/10.1016/j.advwatres.2013.05.005.

26- Robinson, G., Ahmed, A. A., Hamill, G. A., (2016), Experimental Saltwater Intrusion in Coastal Aquifers Using Automated Image Analysis: Applications to Homogeneous Aquifers, Journal of Hydrology 538: 304-13.

27- Watson, T. A., Werner, A. D., Simmons, C. T., (2010), Transience of seawater intrusion in response to sea level rise. Water Resource. Res. 46, W12533, doi:10.1029/2010WR009564 